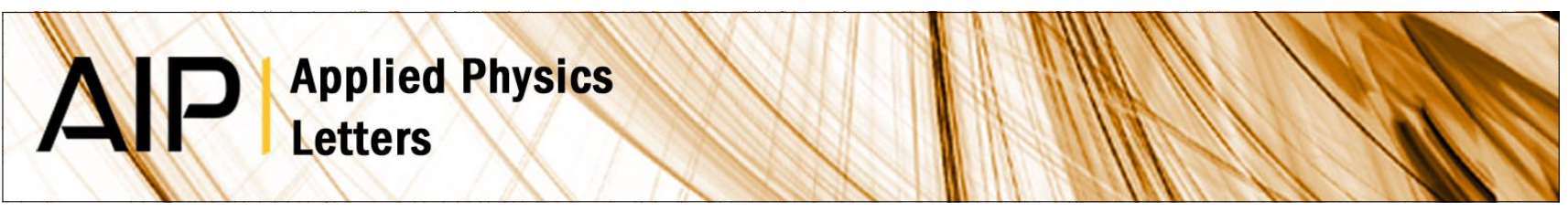

\title{
Energy band bending induced charge accumulation at fullerene/bathocuproine heterojunction interface
}

Shenghao Wang, Takeaki Sakurai, Ryusuke Kuroda, and Katsuhiro Akimoto

Citation: Appl. Phys. Lett. 100, 243301 (2012); doi: 10.1063/1.4728996

View online: http://dx.doi.org/10.1063/1.4728996

View Table of Contents: http://apl.aip.org/resource/1/APPLAB/v100/i24

Published by the American Institute of Physics.

\section{Related Articles}

Effect of metals and annealing on specific contact resistivity of GeTe/metal contacts Appl. Phys. Lett. 101, 012107 (2012)

Methodology of evaluating the influence of the resistance of contact regions in the measurements of sheet resistance on stripes of ultrathin high-resistance materials

Rev. Sci. Instrum. 83, 074701 (2012)

Low resistance $\mathrm{Ti}$ Ohmic contacts to $4 \mathrm{H}-\mathrm{SiC}$ by reducing barrier heights without high temperature annealing Appl. Phys. Lett. 100, 263503 (2012)

Potential barrier increase due to $\mathrm{Gd}$ doping of $\mathrm{BiFeO} 3$ layers in $\mathrm{Nb}$ :SrTiO3-BiFeO3-Pt structures displaying diode-like behavior

Appl. Phys. Lett. 100, 252903 (2012)

Terraces at ohmic contact in $\mathrm{SiC}$ electronics: Structure and electronic states

J. Appl. Phys. 111, 113717 (2012)

\section{Additional information on Appl. Phys. Lett.}

Journal Homepage: http://apl.aip.org/

Journal Information: http://apl.aip.org/about/about_the_journal

Top downloads: http://apl.aip.org/features/most_downloaded

Information for Authors: http://apl.aip.org/authors

\section{ADVERTISEMENT}

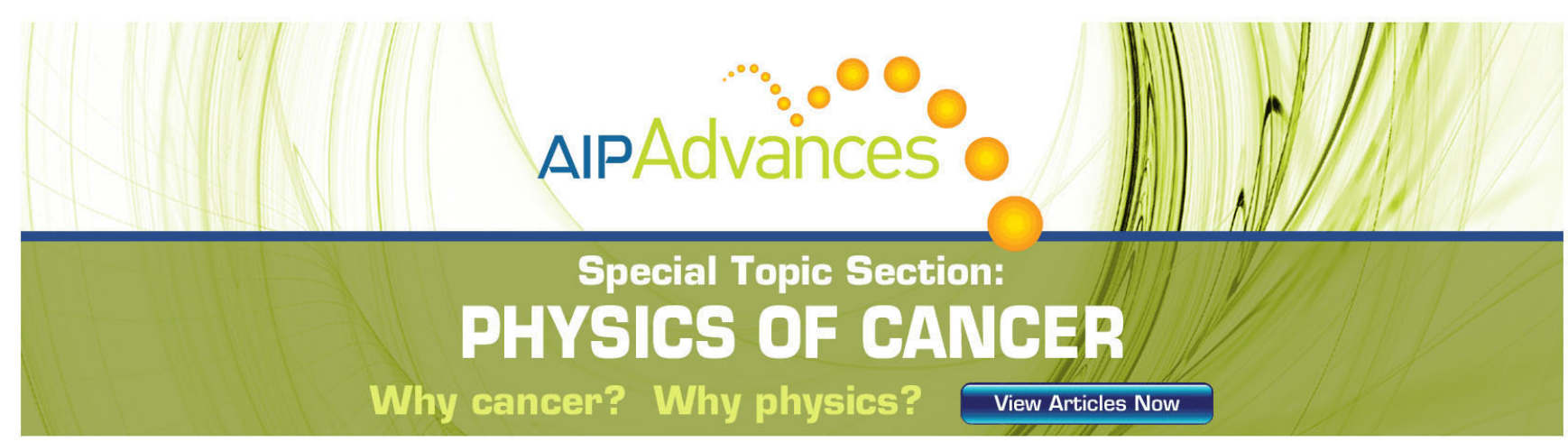




\title{
Energy band bending induced charge accumulation at fullerene/ bathocuproine heterojunction interface
}

\author{
Shenghao Wang, ${ }^{1, a)}$ Takeaki Sakurai, ${ }^{1,2}$ Ryusuke Kuroda, ${ }^{1}$ and Katsuhiro Akimoto ${ }^{1}$ \\ ${ }^{1}$ Institute of Applied Physics, University of Tsukuba, Tennodai 1-1-1, Tsukuba, Ibaraki 305-8573, Japan \\ ${ }^{2}$ PRESTO, Japan Science and Technology Agency (JST), Kawaguchi, Saitama 332-0012, Japan
}

(Received 1 March 2012; accepted 22 May 2012; published online 12 June 2012)

\begin{abstract}
The electronic properties of fullerene $\left(\mathrm{C}_{60}\right)$ /bathocuproine $(\mathrm{BCP}) / \mathrm{Ag}$ heterostructures were studied as a function of the BCP layer thickness by photoemission spectroscopy. For the thin BCP layer, the energy levels are flat and gap states exist at the interface. In contrast, energy band bending occurs at the $\mathrm{C}_{60} / \mathrm{BCP}$ interface when the $\mathrm{BCP}$ layer is thick, resulting in a considerable barrier for electron transport and therefore causing charge accumulation in organic solar cells. The results reveal that a thin BCP layer gives a much more favorable energy level structure and conform that charge accumulation is responsible to the anomalous current-voltage (I-V) curve. (C) 2012 American Institute of Physics. [http://dx.doi.org/10.1063/1.4728996]
\end{abstract}

Organic solar cells (OSCs) have great potential in the photovoltaic market due to the advantages of low cost, easy fabrication, and compatibility with large-scale and flexible substrates by means of low-cost printing and coating technologies. ${ }^{1,2}$ The insertion of a buffer layer between acceptor and top metal electrode can improve the power conversion efficiency of OSCs. ${ }^{3}$ The most commonly used buffer layer is bathocuproine (BCP). Several interpretations have been proposed for the working mechanism of $\mathrm{BCP}$, such as functioning as an exciton-blocking layer, ${ }^{4}$ reducing nonradiative recombination of excitons at the $\mathrm{C}_{60} / \mathrm{Al}$ interface, ${ }^{5}$ increasing the built-in field, ${ }^{6}$ avoiding the formation of aluminum carbide, ${ }^{7}$ and acting as an optical spacer. ${ }^{8}$ However, the exact role of BCP buffer layer in OSCs still remains unclear.

The thicknesses of organic materials strongly affect the performance of OSCs because of the short exciton diffusion length, low carrier mobility, high resistance, and unique carrier hoping mechanism in organic materials. ${ }^{7,9,10}$ It has been demonstrated that a thick BCP buffer layer at accept/cathode layers deteriorates the performance of OSCs. ${ }^{7}$ Wang et al. ${ }^{11}$ recently reported that the shape of current-voltage $(I-V)$ curve of small molecular OSC depends on the BCP layer thickness. They found that the device with thick BCP layer exhibits a kink-like $I$ - $V$ curve (i.e., an anomalous $S$-shaped $I-V$ curve), which results in low fill factor and poor device performance. Several explanations have been proposed for the origin of the $S$-shaped $I-V$ curve, including slow charge carrier transfer at the contacts with electrodes, ${ }^{12}$ the presence of interfacial dipoles, ${ }^{13}$ traps and defects, ${ }^{14}$ and energy barriers at the donor-accept interface. ${ }^{15}$ In Ref. 11, new assumption of charge accumulation was proposed to explain the $S$-shaped $I-V$ curve induced by thick BCP layer. However, there is no report in terms of energy level structure for the cases of thin and thick BCP layers. Furthermore, metal may diffuse toward the active layer $\left(\mathrm{C}_{60}\right)$ through the buffer layer during metal electrode deposition, resulting in undesirable interaction between the active layer and the metal. ${ }^{7,16}$ It is thus critical to study the interfacial properties of $\mathrm{C}_{60} / \mathrm{BCP} /$

${ }^{\text {a)} E l e c t r o n i c ~ m a i l: ~ w s h 8511 @ g m a i l . c o m . ~}$ metal heterostructures as a function of the BCP layer thickness to determine the role of BCP and to clarify the origin of the $\mathrm{S}$-shaped $I-V$ curve. This will enable the performance of OSCs to be improved.

We have reported the electronic properties of $\mathrm{BCP} /$ metal interface. ${ }^{17}$ The present work focuses on the interface of $\mathrm{C}_{60} /$ $\mathrm{BCP}$ heterojunction. We investigated the electronic properties of $\mathrm{C}_{60} / \mathrm{BCP} / \mathrm{Ag}$ heterostructures as a function of the $\mathrm{BCP}$ layer thickness by synchrotron-based in situ ultraviolet photoemission spectroscopy (UPS). We found that the energy level alignment of the heterostructures with thick BCP layer drastically differs from that with thin $\mathrm{BCP}$ layer due to energy band bending.

UPS experiments were performed at beamline BL-3B of the Photon Factory, High Energy Accelerator Research Organization (KEK), Japan. Ex situ cleaned Si (100) wafers were used as substrates. The $\mathrm{C}_{60} / \mathrm{BCP} / \mathrm{Ag}$ heterostructures were formed by depositing $\mathrm{BCP}$ on $\mathrm{Ag}$ and subsequently depositing $\mathrm{C}_{60}$ with increasing thickness from 0.4 to $5 \mathrm{~nm}$ onto BCP/Ag stack layer in a step-by-step way in a deposition chamber $\left(<5.0 \times 10^{-9}\right.$ Torr $)$. BCP layers with thicknesses of 0.8 and $5 \mathrm{~nm}$ were formed in two samples; these represent thin and thick layers, respectively. The nominal thickness of $0.6 \sim 0.8 \mathrm{~nm}$ corresponds to a monolayer of BCP. The $5 \mathrm{~nm}$ BCP layer is sufficiently thick to give a bulklike spectrum and bury the signal of the underlayer without causing any noticeable charging. Prior to deposition, BCP (Wako Corp., sublimated grade) and $\mathrm{C}_{60}$ (Sigma-Aldrich, 99.5\%) were purified three times by vacuum gradient sublimation. The deposition rates for both materials were $0.01 \mathrm{~nm} / \mathrm{s}$, and the thicknesses were monitored by a quartzcrystal microbalance. UPS spectra were measured in an analysis chamber $\left(<9.0 \times 10^{-10}\right.$ Torr $)$ using the photon energy of $30 \mathrm{eV}$. The secondary electron cutoff (SEC) energy was measured under a bias of $-4.0 \mathrm{~V}$.

Figure 1 shows UPS spectra of $\mathrm{C}_{60}$ layers with various thicknesses deposited on BCP $(0.8 \mathrm{~nm}) / \mathrm{Ag}$ stack layer. The abscissa is the electron binding energy $(B E)$ relative to the Fermi level $\left(E_{F}\right)$ of Ag. Fig. 1(b) shows that, apart from the presence of small peak at about $1.8 \mathrm{eV}$, there is hardly any 


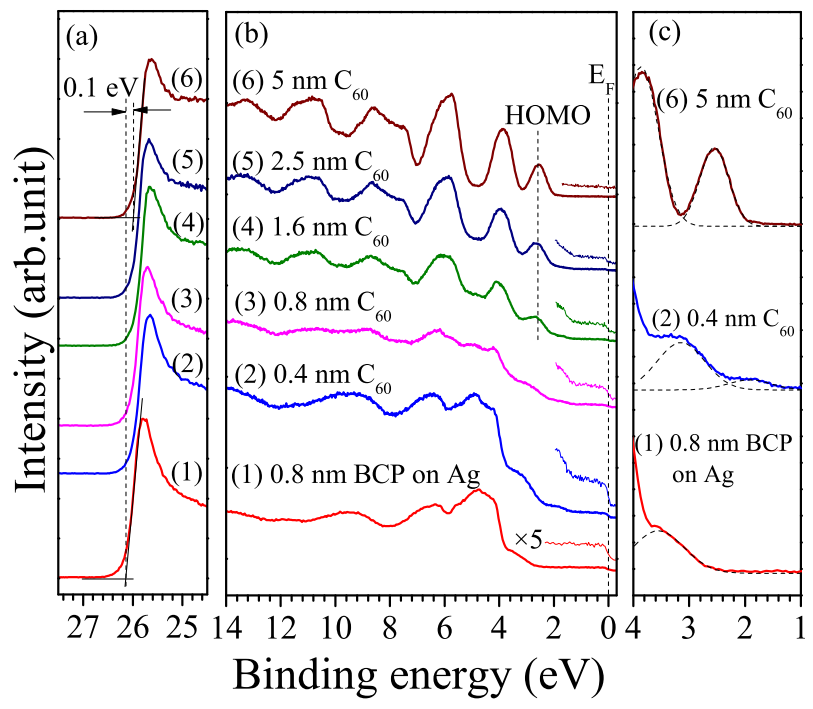

FIG. 1. UPS spectra of $\mathrm{C}_{60}$ layers with various thicknesses deposited on $\mathrm{BCP}(0.8 \mathrm{~nm}) / \mathrm{Ag}$ stack layer at (a) the $S E C$ energy region, (b) the wide region, and (c) the low $B E$ part near the $E_{F}$. (For (c), only spectra of $\mathrm{C}_{60}$ layers with thicknesses of 0.4 and $5 \mathrm{~nm}$ are shown, corresponding to the presence and absence of gap states, respectively. The dashed curves are fitted curves obtained after background subtraction.)

other change and shift for the spectrum after depositing $0.4 \mathrm{~nm} \mathrm{C}_{60}$. As $\mathrm{C}_{60}$ thickness is greater than $0.8 \mathrm{~nm}$, the features of the $\mathrm{BCP}$ underlayer attenuate and the features of the $\mathrm{C}_{60}$ overlayer become discernible. It is difficult to visualize the feature of the highest occupied molecular orbital (HOMO) of $\mathrm{C}_{60}$ for the initial coverage due to spectral overlap with the underlayer features. The HOMO peak of $\mathrm{C}_{60}$, which is clearly discernible at thicknesses above $1.6 \mathrm{~nm}$, exhibits no shift as the thickness is increased up to $5 \mathrm{~nm}$, indicating the pinning of $E_{F}$.

Interestingly, a small peak near $E_{F}$ was clearly observed after depositing $\mathrm{C}_{60}$, as shown in Figs. 1(b) and 1(c). This peak indicates the presence of gap states. With increasing $\mathrm{C}_{60}$ thickness, the peak intensity weakens until it disappears for the $5 \mathrm{~nm} \mathrm{C}_{60}$ layer, indicating that the gap states only exist at the interface. Organic-organic interfaces are expected to be chemically and structurally simple because of the weak interaction of van der Waals inter-molecular forces. Therefore, gap states generally should not be generated at organic-organic interfaces. The observed gap states at the $\mathrm{C}_{60} / \mathrm{BCP}$ interface appear to be generated by the interaction between $\mathrm{C}_{60}$ and $\mathrm{Ag}$ when $\mathrm{Ag}$ diffuses toward the $\mathrm{C}_{60}$ side through the thin $\mathrm{BCP}$ layer or the $\mathrm{C}_{60}$ layer directly contacts with $\mathrm{Ag}$ due to the non-wetting coverage on the thin $\mathrm{BCP}$ layer. To investigate this conjecture, we studied the electronic properties of $\mathrm{C}_{60} / \mathrm{Ag}$ interface by UPS (data not shown); in other word, the case when there is no BCP interlayer. However, no gap states exist at the $\mathrm{C}_{60} / \mathrm{Ag}$ interface in the corresponding $B E$ (just the same as when $\mathrm{BCP}$ layer is present), and the $\mathrm{HOMO}$ peak of $\mathrm{C}_{60}$ on $\mathrm{Ag}$ shows a distinct shift to higher $B E$, which is contradictory to the unchanged HOMO peak of $\mathrm{C}_{60}$ on BCP layer. It has been demonstrated that metal-BCP complex can form during metal contact deposition. ${ }^{4}$ The origin of the gap states at the $\mathrm{C}_{60} / \mathrm{BCP}$ interface can be ascribed to the interaction between $\mathrm{C}_{60}$ and $\mathrm{Ag}-\mathrm{BCP}$ complex, rather than the interaction between $\mathrm{C}_{60}$ and $\mathrm{Ag}$.

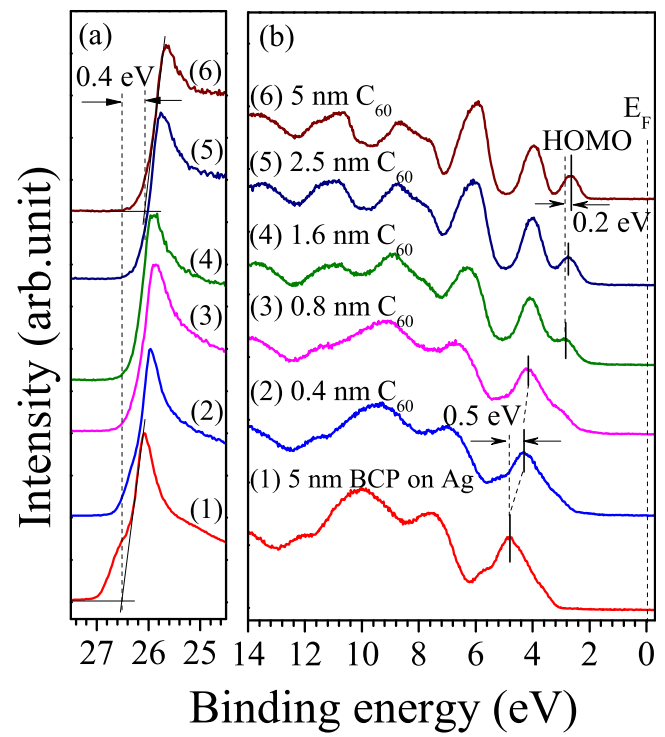

FIG. 2. UPS spectra of $\mathrm{C}_{60}$ layers with various thicknesses deposited on $\mathrm{BCP}(5 \mathrm{~nm}) / \mathrm{Ag}$ stack layer at (a) the $S E C$ energy region and (b) the wide region.

When $\mathrm{BCP}$ is deposited on $\mathrm{Ag}, \mathrm{Ag}$ interacts with $\mathrm{BCP}$, forming $\mathrm{Ag}-\mathrm{BCP}$ complex. $\mathrm{A}_{60} /$ complex interface then forms when $\mathrm{C}_{60}$ is deposited. The electronic properties of $\mathrm{Ag}-\mathrm{BCP}$ complex formed by co-deposition of Ag and $\mathrm{BCP}$ have been studied in our previous study (Ref. 17). We found that the partially filled gap states exist in Ag-BCP complex. This complex facilitates electron transfer along the stacked molecules through adjacent conduction levels. When $\mathrm{C}_{60}$ molecule is chemically bonded to the metal-BCP complex, the energy levels of $\mathrm{C}_{60}$ should be modified, causing shifting, broadening, and the formation of new states. In Fig. 1(a), the shift of the $S E C$ energy, which reflects the vacuum level $(V L)$ change, is just $0.1 \mathrm{eV}$, indicating approximate vacuum level alignment at the $\mathrm{C}_{60} / \mathrm{BCP}$ interface. This occurs for most organic heterojunctions, which can be described by the classical Shockley-Mott model and are characterized by flat energy levels and aligned vacuum levels across the junctions. ${ }^{18}$

Figure 2 shows UPS spectra of $\mathrm{C}_{60}$ layers with various thicknesses deposited on BCP $(5 \mathrm{~nm}) / \mathrm{Ag}$ stack layer. The $\mathrm{C}_{60}$ features are visible for $\mathrm{C}_{60}$ thicknesses up to $1.6 \mathrm{~nm}$. Below this thickness, the spectra are dominated by the BCP features. Significantly, obvious peak shift for the BCP features can be observed upon the deposition of $\mathrm{C}_{60}$, implying that the molecular orbitals of $\mathrm{BCP}$ change. After depositing $0.4 \mathrm{~nm} \mathrm{C}_{60}$, the HOMO peak of BCP (indicated by the short solid line in Fig. 2(b)) abruptly shifts to lower $B E$ by $0.5 \mathrm{eV}$. When the $\mathrm{C}_{60}$ layer thickness is increased to $0.8 \mathrm{~nm}$, the HOMO peak of BCP further shifts by $0.1 \mathrm{eV}$. With further increase of $\mathrm{C}_{60}$ layer thickness, the $\mathrm{BCP}$ features disappear, and the HOMO peak of $\mathrm{C}_{60}$ becomes clear. When the $\mathrm{C}_{60}$ layer thickness is increased from 1.6 to $5 \mathrm{~nm}$, the HOMO peak of $\mathrm{C}_{60}$ shifts to lower $B E$ by $0.2 \mathrm{eV}$. The change of the HOMO peak indicates that band bending occurs for both $\mathrm{BCP}$ and $\mathrm{C}_{60}$ at the interface of $\mathrm{C}_{60} / \mathrm{BCP}$ heterojunction.

In Fig. 2(b), there is no new peak near the $E_{F}$ after $\mathrm{C}_{60}$ deposition, indicating that there are no gap states at the interface. Fig. 2(a) shows that the SEC energy shifts to lower $B E$ 
(a)

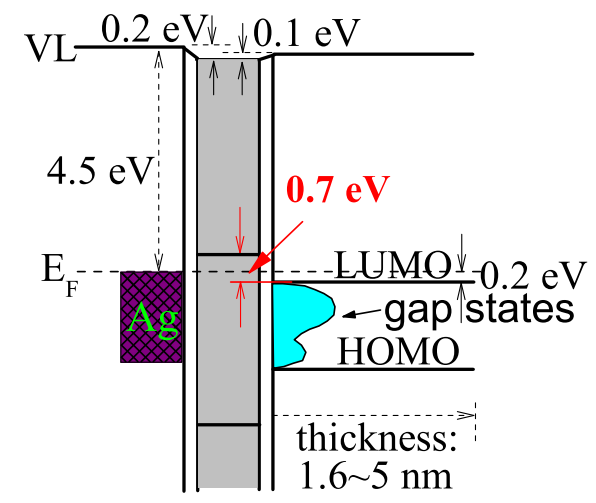

Complex $\quad \mathrm{C}_{60}$ (b)

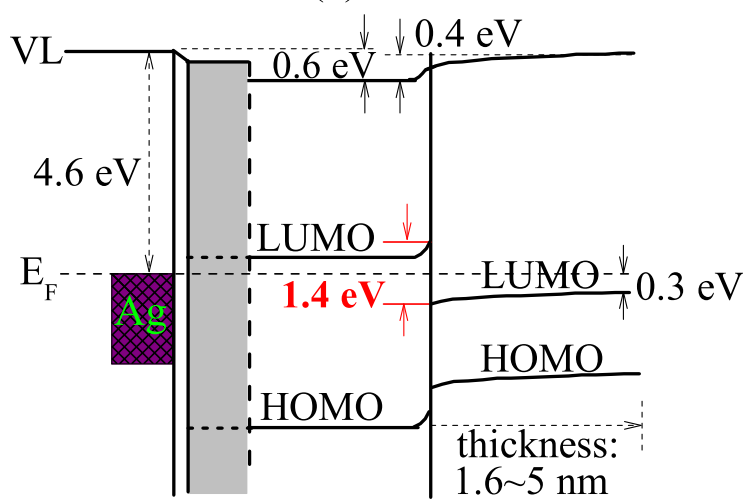

Complex $\mathrm{BCP} \quad \mathrm{C}_{60}$

FIG. 3. Schematic energy level diagrams of $\mathrm{C}_{60} / \mathrm{BCP} / \mathrm{Ag}$ heterostructures formed by depositing $\mathrm{C}_{60}$ layers with increasing thickness on (a) 0.8 nm $\mathrm{BCP}$ and (b) $5 \mathrm{~nm} \mathrm{BCP}$ on $\mathrm{Ag}$.

by $0.4 \mathrm{eV}$ with increasing $\mathrm{C}_{60}$ thickness. As compared to the thin BCP layer case, the $V L$ shift is much larger. This implies the breakdown of the vacuum level alignment rule and the formation of interface dipole layer. The origin of this dipole layer will be discussed later.

Figure 3 shows energy level diagrams of the $\mathrm{C}_{60} / \mathrm{BCP} /$ $\mathrm{Ag}$ heterostructures. The HOMO level was determined by linearly extrapolating the HOMO peak. The lowest unoccupied molecular orbital (LUMO) level was derived via the optical band gaps of 3.5 (Ref. 19) and $1.7 \mathrm{eV}$ (Ref. 20) for BCP and $\mathrm{C}_{60}$, respectively. For the thin BCP layer (Fig. 3(a)), the molecular orbitals of $\mathrm{C}_{60}$ and $\mathrm{BCP}$ do not change. The interaction between $\mathrm{Ag}-\mathrm{BCP}$ complex and $\mathrm{C}_{60}$ may drive the equivalence of the LUMO levels and thus give rise to the flat energy levels. The induced gap states at the interface may facilitate electron transfer in OSCs. For the thick BCP layer (Fig. 3(b)), the nominal BCP layer actually consists of two layers: the Ag-BCP complex sublayer formed during the initial deposition of $\mathrm{BCP}$, and a dominant $\mathrm{BCP}$ layer formed during the continuous deposition of BCP till $5 \mathrm{~nm}$. The thick $\mathrm{BCP}$ layer inhibits the formation of $\mathrm{C}_{60} /$ complex interface. In this case, we can observe energy band bending at the $\mathrm{C}_{60}$ / $\mathrm{BCP}$ interface, which is associated with a balance of charges in the space charge regions on both sides of the $\mathrm{C}_{60} / \mathrm{BCP}$ heterojunction.

Based on the charge neutrality level (CNL) model reported by Vázquez et al., ${ }^{21}$ the $C N L$ represents the electronegativity of the molecule to some degree and a deeper $C N L$ means a strong attraction for electrons. The CNLs of BCP and $\mathrm{C}_{60}$ have been reported to be 3.8 (Ref. 22) and $4.6 \mathrm{eV},{ }^{22,23}$ respectively. When forming $\mathrm{C}_{60} / \mathrm{BCP}$ interface, charge redistributes across the interface due to the much deeper $C N L$ of $\mathrm{C}_{60}$. This charge redistribution induces band bending and dipole layer formation. From the CNL model, the magnitude of the resulting interface dipole was derived to be $-0.37 \mathrm{eV}$, being consistent with the experimental value of $0.4 \mathrm{eV}$. The minus sign indicates that the direction of electron transfer is from $\mathrm{BCP}$ to $\mathrm{C}_{60}$.

In Fig. 3, we can see that the LUMO level of $\mathrm{C}_{60}$ in the thick BCP layer case is deeper than that of the thin $\mathrm{BCP}$ layer case, especially at the interface. The energy band bend- ing of both BCP and $\mathrm{C}_{60}$ and the much deeper LUMO level of $\mathrm{C}_{60}$ enlarge the energy difference of LUMO levels between $\mathrm{C}_{60}$ and $\mathrm{BCP}$ at the interface. This energy difference is $1.4 \mathrm{eV}(0.7 \mathrm{eV})$ for the thick (thin) BCP layer. The LUMO offset of the $\mathrm{BCP} / \mathrm{C}_{60}$ interface based on the $\mathrm{BCP} / \mathrm{C}_{60} /$ copper phthalocyanine/indium tin oxide heterostructures has been reported to be as large as $2 \mathrm{eV},{ }^{24}$ which is comparable with that of the present study. This suggests that the interfacial electronic properties between $\mathrm{C}_{60}$ and $\mathrm{BCP}$ are independent on the deposition sequence. The high energy offset between the LUMO of $\mathrm{C}_{60}$ and the LUMO of BCP at the interface will act as a distinct barrier for electron extraction from $\mathrm{C}_{60}$ to the electrode when the dissociated excitons move to the $\mathrm{C}_{60}$ /BCP interface in OSCs. This will cause electrons accumulation on $\mathrm{C}_{60}$ side, which may induce the anomalous Sshaped $I-V$ curve.

In conclusion, the electronic properties of $\mathrm{C}_{60} / \mathrm{BCP} / \mathrm{Ag}$ heterostructures are strongly dependent on the thickness of the $\mathrm{BCP}$ layer. When the $\mathrm{BCP}$ layer is thin, $\mathrm{C}_{60} /$ complex interface is formed, and the interaction between them results in gap states at the interface, which are beneficial to the OSCs. When the BCP layer is thick, energy band bending occurs at the $\mathrm{C}_{60} / \mathrm{BCP}$ interface. It generates a considerable barrier for electrons transport (as high as $1.4 \mathrm{eV}$ ). This will induce charge accumulation at the $\mathrm{C}_{60} / \mathrm{BCP}$ interface in OSCs, which is undesirable for the device performance. The results demonstrate that a thin BCP layer gives a much more favorable energy level alignment for OSCs and also offer direct support for the conjecture that charge accumulation is responsible to the anomalous $S$-shaped $I-V$ characteristics of OSCs.

This work was partially supported by JSPS, and the first author additionally appreciates the support of China Scholarship Council (CSC) Oversea Study Program.

${ }^{1}$ P. Peumans, A. Yakimov, and S. R. Forrest, J. Appl. Phys. 93, 3693 (2003).

${ }^{2}$ M. D. Wang, F. Y. Xie, W. Q. Xie, S. Z. Zheng, N. Ke, J. Chen, N. Zhao, and J. B. Xu, Appl. Phys. Lett. 98, 183304 (2011).

${ }^{3}$ N. N. Wang, J. S. Yu, Y. Zang, J. Huang, and Y. D. Jiang, Sol. Energy Mater. Sol. Cells 94, 263 (2010). 
${ }^{4}$ P. Peumans, V. Bulovic, and S. R. Forrest, Appl. Phys. Lett. 76, 2650 (2000).

${ }^{5}$ M. Vogel, S. Doka, Ch. Breyer, M. Ch. Lux-Steiner, and K. Fostiropoulos, Appl. Phys. Lett. 89, 163501 (2006).

${ }^{6}$ Q. L. Song, C. M. Li, M. L. Wang, X. Y. Sun, and X. Y. Hou, Appl. Phys. Lett. 90, 071109 (2007).

${ }^{7}$ C. C. Chang, C. F. Lin, J. M. Chiou, T. H. Ho, Y. Tai, J. H. Lee, Y. F. Chen, J. K. Wang, L. C. Chen, and K. H. Chen, Appl. Phys. Lett. 96, 263506 (2010).

${ }^{8}$ B. P. Rand, J. Li, J. Xue, R. J. Holmes, M. E. Thompson, and S. R. Forrest, Adv. Mater. 17, 2714 (2005).

${ }^{9}$ Y. Terao, H. Sasabe, and C. Adachi, Appl. Phys. Lett. 90, 103515 (2007).

${ }^{10}$ M. A. Khan, W. Xu, Khizar-ul-Haq, X. W.Zhang, Y.Bai, X. Y.Jiang, Z. L.Zhang, and W. Q.Zhu, J. Phys. D: Appl. Phys. 41, 225105 (2008).

${ }^{11}$ J. C. Wang, X. C. Rena, S. Q. Shi, C. W. Leung, and P. K. L. Chan, Org. Electron. 12, 880 (2011).

${ }^{12}$ M. Glatthaar, M. K. Riede, N. Keegan, K. Sylvester-Hvid, B. Zimmermann, M. Niggemann, A. Hinsch, and A. Gombert, Sol. Energy Mater. Sol. Cells 91, 390 (2007).

${ }^{13}$ A. Wagenpfahl, D. Rauh, M. Binder, C. Deibel, and V. Dyakonov, Phys. Rev. B 82, 115306 (2010).
${ }^{14}$ A. Kumar, S. Sista, and Y. Yang, J. Appl. Phys. 105, 094512 (2009).

${ }^{15}$ C. Uhrich, D. Wynands, S. Olthof, M. K. Riede, K. Leo, S. Sonntag, B. Maennig, and M. Pfeiffer, J. Appl. Phys. 104, 043107 (2008).

${ }^{16}$ J. Hwang, A. Wan, and A. Kahn, Mat. Sci. Eng. R 64, 23 (2009).

${ }^{17}$ T. Sakurai, S. Toyoshima, H. Kitazume, S. Masuda, H. Kato, and K. Akimoto, J. Appl. Phys. 107, 043707 (2010).

${ }^{18}$ T. W. Ng, M. F. Lo, S. T. Lee, and C. S. Lee, Appl. Phys. Lett. 100, 113301 (2012).

${ }^{19}$ S. Toyoshima, K. Kuwabara, T. Sakurai, T. Taima, K. Saito, H. Kato, and K. Akimoto, Jpn. J. Appl. Phys. 46, 2692 (2007).

${ }^{20}$ Y. Wang, J. M. Holden, A. M. Rao, W. Lee, X. X. Bi, S. L. Ren, G. W. Lehman, G. T. Hager, and P. C. Eklund, Phys. Rev. B 45, 14396 (1992).

${ }^{21}$ H. Vázquez, R. Oszwaldowski, P. Pou, J. Ortega, R. Perez, F. Flores, and A. Kahn, Europhys. Lett. 65, 802 (2004).

${ }^{22}$ A. Kahn, W. Zhao, W. Y. Gao, H. Vazquez, and F. Flores, Chem. Phys. 325, 129 (2006).

${ }^{23}$ W. Zhao and A. Kahn, J. Appl. Phys. 105, 123711 (2009).

${ }^{24}$ J. X. Tang, Y. C. Zhou, Z. T. Liu, C. S. Lee, and S. T. Lee, Appl. Phys. Lett. 93, 043512 (2008). 